\title{
Mecanismo de fissuraçao sob tração direta de compósitos cimentícios reforçados com fibras curtas de sisal
}

Paulo Roberto Lopes Lima ${ }^{1}$

Romildo Dias Toledo Filho ${ }^{2}$

José Mario Feitosa Lima ${ }^{3}$

Resumo: Embora a utilização de fibras vegetais como reforço de matrizes a base de cimento remonte à década de 70, poucos estudos têm sido realizados para investigação experimental e analítica desses compósitos sob tração direta. Nesse trabalho, foi avaliado o comportamento de argamassas reforçadas com fibras curtas de sisal sob ensaio de tração. O diagrama carga-deslocamento foi expresso em termos de tensão-deformação até a abertura da fissura e, após esse ponto, em termos de tensão-abertura de fissura. Comparados com a matriz, que apresentou ruptura frágil, os compósitos reforçados com 1\%, 2\% e 3\% de fibras de sisal apresentaram propagação da fissura a grandes deslocamentos com manutenção de uma resistência residual. Modelos analíticos baseados na Mecânica da Fratura foram utilizados para determinaçáo da tenacidade de fratura e comprimento característico do material e demonstraram que a adiçáo de fibra aumenta a capacidade de absorção de energia do material.

Palavras-Chave: mecânica da fratura;argamassa; fibras vegetais

Abstract:Although the use of vegetables fibers as reinforcement in cement based matrices goes back to the 70 s, few studies have been performed for analytical and experimental investigation of these composites in direct tensile. In this study, It was evaluated the tensile behavior of composite mortars reinforced with 1\%, $2 \%$ and $3 \%$ of short sisal fibers. The softening curve was represented by stress-strain diagram until first crack and, after this point, it was represented by stress-crack opening diagram. Compared with the matrix, which showed brittle fracture, the composites showed crack propagation to large displacements with maintenance of residual stress. Analytical models based Fracture Mechanics were used to determine fracture energy and characteristic length of material and the results showed that presence of fibers increment the toughness of cement based mortar under tensile.

Keywords: fracture mechanics; mortar; vegetable fibers

\footnotetext{
1 Programa de Pós-graduação em Engenharia Civil e Ambiental - PPGECEA, Universidade Estadual de Feira de Santana UEFS, Av. Transnordestina, SN, Novo Horizonte, Feira de Santana, BA, 44030-900, Brasil. E-mail: lima.prl@pq.cnpq.br.

2 Programa de Pós-graduação em Engenharia Civil - PEC-COPPE, Universidade Federal do Rio de Janeiro. Centro de Tecnologia, Bloco B, Ilha do Fundão, Rio de Janeiro, RJ. E-mail: toledo@coc.ufrj.br.

3 Programa de Pós-graduaçáo em Engenharia Civil e Ambiental - PPGECEA, Universidade Estadual de Feira de Santana UEFS, Av. Transnordestina, SN, Novo Horizonte, Feira de Santana, BA, 44030-900, Brasil. E-mail: lima.jmf@gmail.com.
} 


\section{INTRODUÇÃO}

A construção civil é um dos setores industriais com maior impacto ambiental. A nível mundial, as atividades relacionadas a construção de edificações consomem $24 \%$ de todo o recurso natural extraído do planeta (Bribián et al, 2011). $\mathrm{Na}$ Europa, tem sido estimado que as construçóes consumem cerca de $40 \%$ da energia, produzem $30 \%$ das emissóes de dióxido de carbono e geram $40 \%$ dos resíduos sólidos (Norton e Skates, 2000). Devido a isso tem crescido o interesse em materiais e elementos construtivos que incorporem conceitos de sustentabilidade, ou seja, que sejam biodegradáveis e renováveis. Sob esse aspecto, a utilização das fibras vegetais na produção de fibrocimentos apresenta uma larga vantagem em relação às fibras poliméricas, como PVA, que têm sido utilizadas atualmente em substituição à fibra de asbesto, reconhecidamente cancerígena.

A utilização de fibras vegetais, como sisal e coco, para reforçar matrizes frágeis a base de cimento é uma técnica que foi amplamente utilizada na década de 70 , na produçáo de placas planas e corrugadas para elementos de cobertura em países em desenvolvimento (Coutts, 1988). No inicio dos anos 80, o uso de fibras vegetais como reforço de compósitos a base de cimento foi bruscamente interrompido depois que alguns elementos construtivos apresentaram perda de resistência após poucos meses de uso. Somente após a identificação dos mecanismos de deterioração da fibra vegetal em meio alcalino (Gram, 1983) e da proposição de soluçóes para garantia de compósitos duráveis (Toledo Filho et al, 2003; Lima e Toledo Filho, 2008), que coincidiram com a preocupação mundial sobre sustentabilidade, é que a utilizaçáo deste tipo de fibra como reforço de compósitos a base de cimento voltou a despertar interesse cientifico.

A produção de elementos construtivos com esse material, no entanto, ainda é incipiente, principalmente porque, para que se possa dimensionar tais elementos de forma segura e econômica, é preciso avaliar e predizer com precisão o seu comportamento mecânico, notadamente sob cargas de tração e flexão.

A utilização de fibras curtas e distribuídas aleatoriamente em matrizes a base de cimento foi iniciada de forma sistemática com a mistura de fibras de aço em concretos, com o objetivo de melhorar a capacidade de absorçáo de energia do material. Após avanços na produçáo de matrizes e o melhor entendimento dos mecanismos de transferências de tensôes, notadamente nas últimas 5 décadas, foi possível alcançar, mesmo com fibras curtas, compósitos cimentícios com elevado desempenho. Denominados de "High Performance Fiber Reinfoced Cement Composites (HPFRCC)" ou "Ultra High Performance Cement Composites (UHP-FRC)", esses compósitos reforçados com fibras curtas de aço apresentavam aumento de tensão de tração mesmo após a fissuração da matriz (Naaman, 2012). Esse comportamento, denominado "strain-hardening", é apresentado de forma simplificada na curva (a) da Figura 1, e se caracteriza pelo surgimento de múltiplas fissuras na matriz. Mesmo utilizando fibra polimérica, comportamento similar foi obtido por Li et al (1995), em um compósito denominado ECC ("Engineered Cementitious Composites"), que apresenta um pequeno aumento da resistência após a primeira fissura, e somente após atingir grandes deformaçóes, em um comportamento denominado pelos autores de "pseudo strain hardening” e representado pela curva (b) da Figura 1. Comportamento similar foi obtido recentemente em compósitos reforçados com fibras curtas de sisal (Ferreira, 2012) após a utilização de matrizes de cimento com reologia adequada.

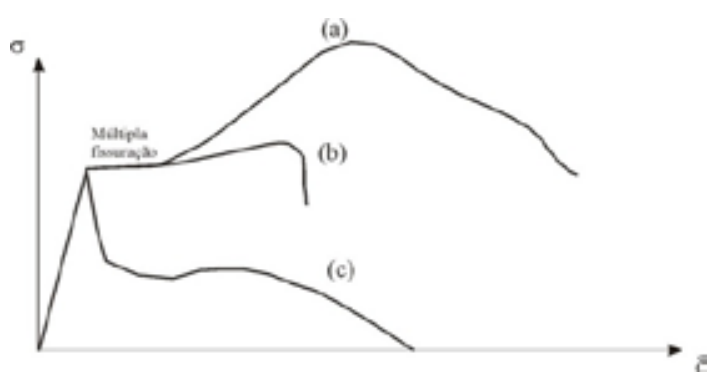

Figura 1 - Curvas esquemáticas do comportamento de compósitos tracionados

Apesar dessa evolução para compósitos de maior desempenho, a maioria dos compósitos reforçados com fibras curtas utilizados atualmente, como o concreto reforçado com fibras de aço, tem como objetivo aumentar a resistência ao impacto e aumentar a deformaçáo de ruptura e, assim, contribuir para melhoria da ductilidade do material sob compressão, flexão e torção (CCI, 2010). 
Para esses compósitos convencionais, o comportamento sobre tração direta é definido pela redução da tensão após a primeira fissura (denominado "strain softening") e manutenção de um valor residual de tensão a grandes deformaçóes, como mostra a curva (c) da Figura 1. A intensidade da tensão residual depende de vários fatores como tipo de fibra, volume de fibra e matriz. A deformação máxima nesses compósitos é usualmente atingida quando as fibras são arrancadas da matriz. Os compósitos produzidos com fibras vegetais randomicamente distribuídas na matriz cimentícia convencional apresenta esse comportamento, devido ao menor módulo de elasticidade da fibra, comparado à matriz de cimento, e baixa tensão de aderência fibra-matriz (Lima, 2004). O entendimento dos mecanismos que afetam esse comportamento é fundamental para que melhorias possam ser implementadas nesse tipo de compósito, e assim contribuindo para que fibras vegetais possam ser utilizadas com maior intensidade na produção de elementos construtivos de fibrocimento.

Alguns estudos (Visalvanichm e Naaman, 1983; Stang et al, 1995) foram realizados com o objetivo de desenvolver a relação tensão-abertura de fissura para compósitos tracionados que apresentam perda de resistência após a fissuração da matriz. Baseados em resultados experimentais para compósitos com fibras curtas de aço, não há uma validaçáo dos conceitos estabelecidos em compósitos reforçados com fibras curtas de baixo módulo, como as fibras de sisal, por exemplo. Este trabalho tem como objetivo avaliar a influência da adição de 1, 2 e 3\% de fibras de sisal, com comprimento de $25 \mathrm{~mm}$, sobre o comportamento mecânico de compósitos a base de cimento submetidos a esforços de tração direta. A partir de modelos de microfissuração baseados na Mecânica da Fratura foram determinados valores de tenacidade de fratura e comprimento característico do material que permitiram avaliar a ductilidade do material com a adição de fibras de sisal.

\section{MATERIAIS E MÉTODOS}

\section{MATERIAIS}

As fibras de sisal utilizadas no presente estudo foram produzidas no município de Valente, Bahia. Elas foram lavadas em água quente $\left(50^{\circ}\right.$
C), para remoção das graxas e impurezas superficiais que afetam a hidrataçáo do cimento, alinhadas em uma escova de aço para separação das fibras individuais e cortadas com $25 \mathrm{~mm}$ de comprimento. Foram utilizados volumes de adição de $1 \%, 2 \%$ e $3 \%$. As fibras de sisal foram largamente caracterizadas (Toledo Filho, 1997) e tem resistência à tração média de $577 \mathrm{MPa}$ e módulo de elasticidade de cerca de $19 \mathrm{GPa}$.

A matriz utilizada foi uma argamassa de cimento Portland com traço, em massa, de 1:1 (cimento:areia) e fator água-cimento de 0,4. Empregou-se cimento Votoran CP III - 40, com densidade de $3,0 \mathrm{~g} / \mathrm{cm}^{3}$ e área superficial medida por fisissorcão (BET multiponto, com $\mathrm{N}_{2}$ ) de $0,2974 \mathrm{~m}^{2} / \mathrm{g}$. e areia quartzosa de rio com módulo de finura 2,37 e massa específica de $2,64 \mathrm{~g} / \mathrm{cm}^{3}$, disponível comercialmente.

As misturas foram produzidas em argamassadeira de $20 \mathrm{dm}^{3}$. Após o lançamento do cimento, areia e parte da água, as fibras foram sendo lançadas manualmente e de forma gradativa, com a argamassadeira em movimento. Após o lançamento de toda a fibra, o restante da água foi adicionado.

A mistura foi então lançada sobre moldes metálicos de dimensóes $100 \mathrm{~mm}$ x $400 \mathrm{~mm}$, formando uma placa de $15 \mathrm{~mm}$ de espessura. Durante o lançamento foi realizada vibraçáo externa com mesa vibratória. Após 24 horas da moldagem, as amostras foram levadas para câmara úmida, com umidade relativa acima de $95 \%$, para cura durante 28 dias.

\section{MÉTODO DE ENSAIO}

\section{Ensaio de traçáo direta}

Após a cura, as placas foram cortadas com serra adiamantada para produzir 4 corpos de prova de dimensôes $50 \mathrm{~mm}$ x $200 \mathrm{~mm}$, para realização do ensaio de tração direta.

O ensaio de traçáo direta foi realizado em uma máquina Shimadzu $1000 \mathrm{kN}$, servocontrolada, com faixa de carregamento de $20 \mathrm{kN}$ e com controle de deslocamento do travessão da máquina de ensaio, a uma taxa de $0,1 \mathrm{~mm} / \mathrm{min}$.

As deformaçôes foram medida de duas formas. Até o surgimento da primeira fissura a amostra encontra-se integra e o comportamento tensão-deformação pode ser descrito pela Mecâ- 
nica do Contínuo. As deformações são então medidas por extensômetros elétricos ("strain gages") com $70 \mathrm{~mm}$ de comprimento, colados no terço central de ambos os lados da amostra, como mostra a Figura 2. Após o surgimento da fissura, a Mecânica do Contínuo não é mais aplicável e a deformação perde o significado. O comportamento do compósito é entâo regido pela abertura da fissura à medida que o deslocamento do travessão da máquina de ensaio é aplicado, sendo mais bem descrito pela Mecânica da Fratura. Dessa forma, o diagrama tensão-deslocamento é interpretado como um diagrama tensão-abertura de fissura, obtido admitindo que a abertura inicial corresponde ao deslocamento quando a tensão atinge o pico.

Para minimizar o efeito de um possível desalinhamento do corpo de prova foi criado um dispositivo com rótulas esféricas, e para evitar o esmagamento pelas garras da máquina de ensaio as amostras foram coladas com adesivo epóxi a elementos de ligação metálicos, como mostra a Figura 2.
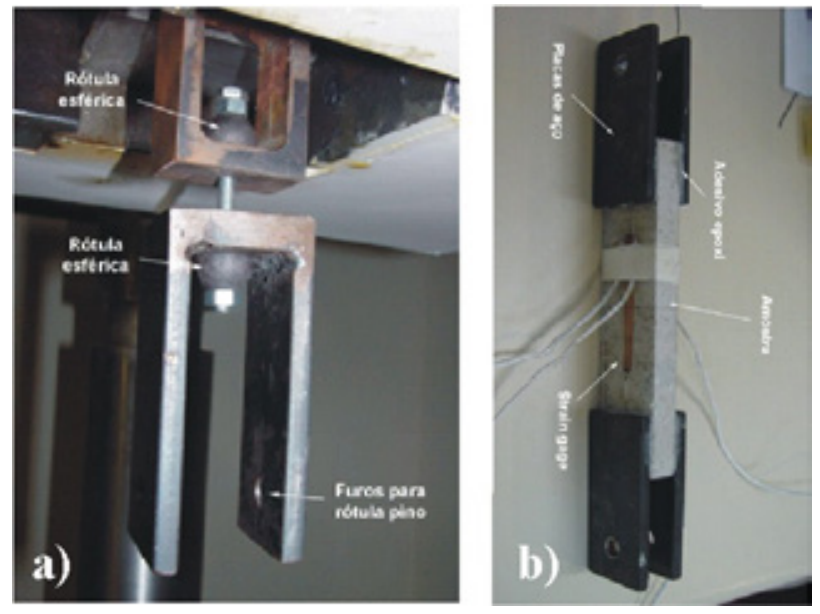

Figura 2. Configuração do ensaio de tração direta: a) sistema de ligação com maquina de ensaio; b) amostra com instrumentação

\section{Ensaio de compressáo axial}

Foram moldadas amostras para realização de ensaios de compressáo, que serviram como referência. A moldagem foi realizada em corpos de prova cilíndricos, com $50 \mathrm{~mm}$ de diâmetro e 100 mm de altura, em três camadas com adensamento externo por mesa vibratória.

Os ensaios de compressão foram realizados na mesma máquina de ensaio com taxa de defor- mação axial de $0,05 \mathrm{~mm} / \mathrm{min}$. Os deslocamentos axiais foram medidos por dois extensômetros tipo LVDT, posicionados na regiáo central da amostra. Foram determinadas a resistência à compressão (fc) e o módulo de elasticidade (Ec), de acordo com a norma ASTM C469/02.

\section{RESULTADOS E DISCUSSÁO}

\section{MECANISMO DE FISSURAÇÃO}

Na Figura 3 são apresentadas curvas típicas obtidas para cada mistura estudada. Até a carga de pico (correspondente à resistência à tração) o deslocamento da amostra é representado pela deformação axial $\varepsilon$. Após a carga de pico, o deslocamento é representado pela abertura de fissura w.

Inicialmente o comportamento do material sob traçáo é formado por um ramo linear elástico que caracteriza o material íntegro, apenas com microfissuras ao redor dos grãos do agregado devido a retraçóes nas primeiras idades. Com o aumento da tensão as microfissuras tendem a se propagar pela pasta de cimento, interligando-se, e resultando em uma leve náo linearidade no diagrama tensão-deformação.

Durante o desenvolvimento dessas microfissuras a teoria do continuo homogêneo é ainda aplicável para o material porque as microfissuras estão no mesmo nível de escala da heterogeneidade da argamassa (Vonk, 1993). Quando as macrofissuras começam a se desenvolver, o nível de escala das fissuras rapidamente ultrapassa o nível de escala da estrutura e a teoria do continuo não é mais aplicável, ou seja, o conceito de deformação perde o seu sentido. A partir da tensão de pico, as fissuras tem que ser levadas em consideraçáo como descontinuidades que, junto com o contínuo, determinam o comportamento da estrutura feita com esse material.

Utilizando os conceitos da Mecânica da Fratura aplicados ao concreto, e demais materiais cimentícios, o comportamento sob tração após a abertura da fissura é modelado considerando que na seção fissurada há duas zonas distintas: uma zona livre de tensóes, que corresponde a uma fissura aberta, e uma zona de fissuração fictícia, localizada na raiz da fissura e que ainda consegue transferir tensóes. A Figura 4a apresenta de forma esquemática essas regiôes. 

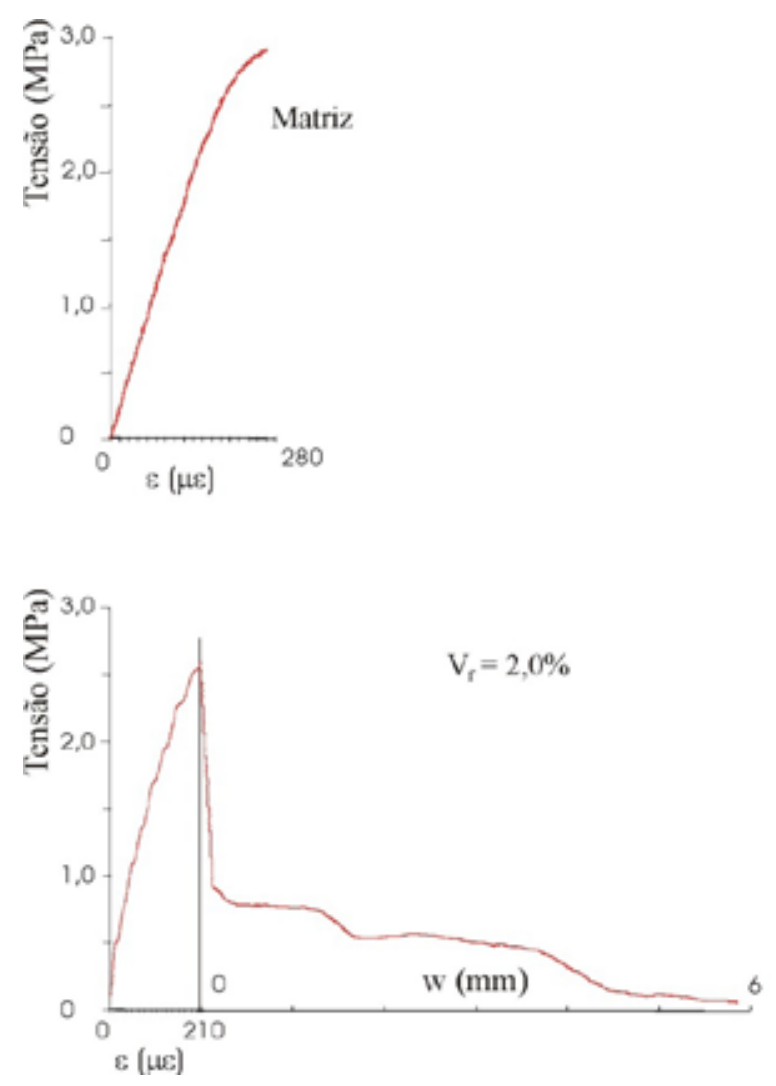
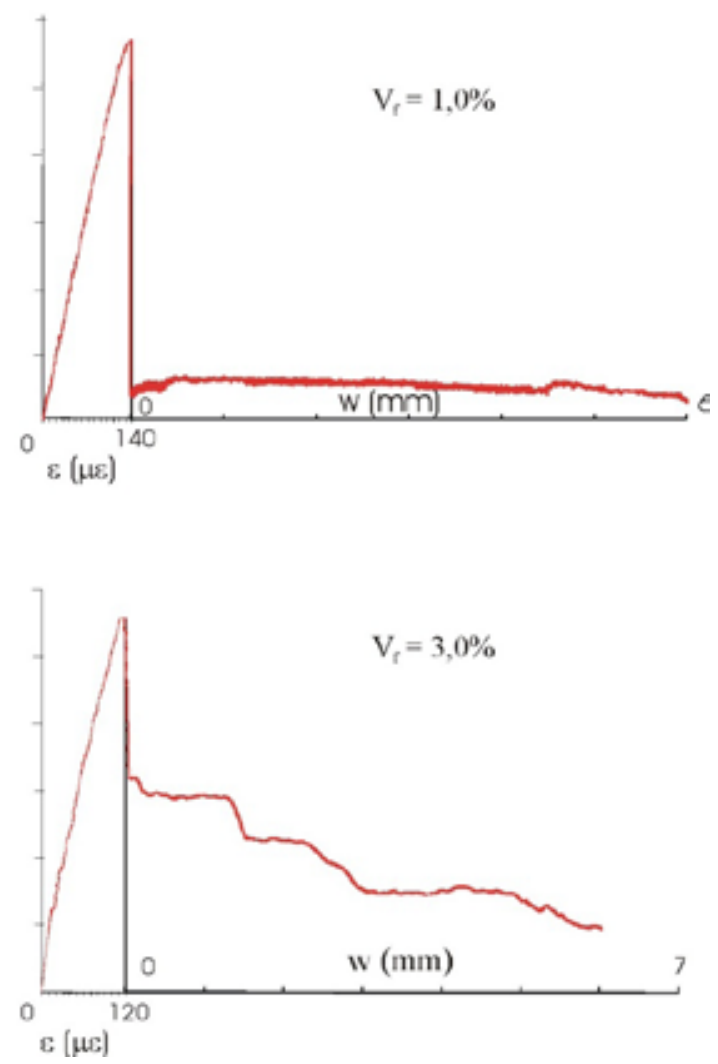

Figura 3 - Curva tensão-deformação $(\varepsilon)$ e tensão-abertura de fissura (w) para matriz e compósitos sob tração direta

A zona de fissuração fictícia é formada por uma parte onde os agregados cruzam a fissura e por uma parte em que há uma microfissuração em formação, denominada zona de processo (RILEM, 2002). Este modelo, denominado Modelo de Fissura Fictícia (MFF), ou Modelo de Fissura Coesiva, foi proposto em 1976 por Hillerborg e colaboradores (Van Mier, 1997) e indica que, apesar de ser assumido que a fissura do concreto sob tração inicia tão logo sua resistência à tração seja atingida, esta fissura não é imediatamente livre de tensões, mas mantém certa capacidade de transmissão de esforços perpendicularmente às faces da fissura. De acordo com Ghebrab e Soroushian (2011), no caso de pastas de cimento, a principal contribuição para manutenção da resistência é o arrancamento de cristais de hidróxido de cálcio $(\mathrm{CH})$ e no caso de argamassas uma contribuiçáo adicional é resultante da energia necessária para o arrancamento dos grãos de areia que são envolvidos pela pasta de cimento.

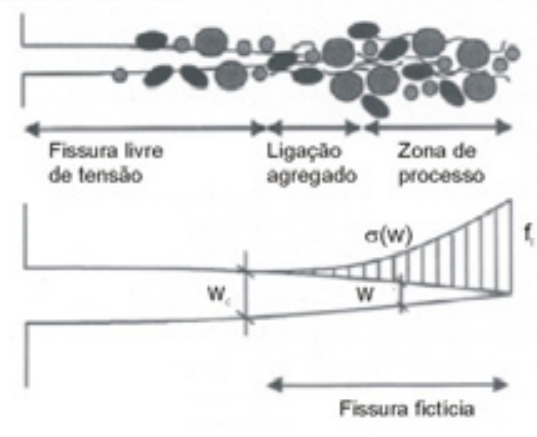

a)

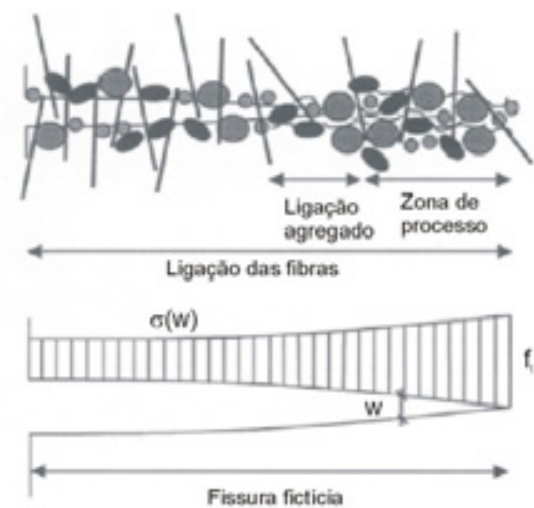

b)

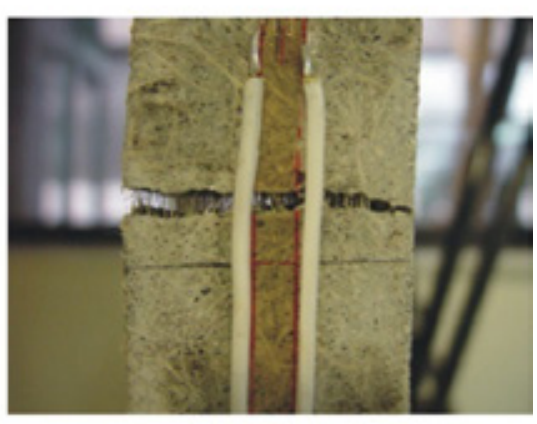

c)

Figura 4 Transferência de tensôes: a) em concreto fissurado de acordo com Modelo de Fissura Fictícia (RILEM, 2002); b) em compósito fissurado (RILEM, 2002); c) verificação experimental da ligação das fibras na fissura. 
Para compósitos, a presença das fibras cruzando as fissura contribui para essa capacidade de transmissão de esforços não apenas na fissura fictícia mas também na fissura real, como mostram as partes (b) e (c) da Figura 4, o que reduz a velocidade de propagaçáo da fissura e permite certa manutençáo de resistência, mesmo a grandes deslocamentos.

Ao possibilitar a transferência de tensões de forma contínua, à medida que a deformação vai sendo desenvolvida, a presença das fibras modifica o comportamento do material que continua, dessa forma, sendo regido também pela Mecânica do Contínuo. De fato, de acordo com o modelo apresentado por Stanget al (1995), a tensão pós-fissuração dos compósitos com fibras é composta por três componentes que representam a contribuição da matriz fissurada, a contribuição da fibra cruzando a fissura e a contribuição elástica da fibra quando do surgimento da primeira fissura.

Tanto na matriz quanto nos compósitos estudados neste trabalho a falha foi governada pela formação e propagação de uma fissura única. A Figura 5 apresenta os corpos de prova de todas as misturas após a ruptura. A maioria das amostras fissurou próximo ao centro do comprimento, ainda que, devido à seção constante das amostras, a localização da primeira fissura é definida por uma fragilidade isolada, como um vazio, em um ponto qualquer do corpo de prova.

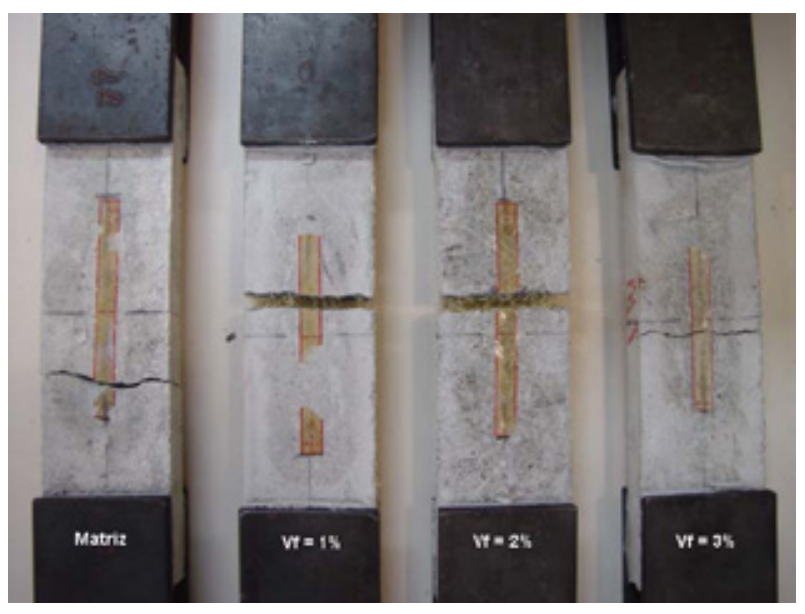

Figura 5 - Forma de ruptura típica da matriz e compósitos reforçados com fibras curtas de sisal sob tração

\section{MODELAGEM DO COMPORTAMENTO TENSÃO-ABERTURA DE FISSURA}

De acordo com o MFF o comportamento mecânico da fissura fictícia é caracterizado pela relação tensão-abertura de fissura, $\sigma(w)$, que é usualmente assumida como decrescente à medida que aumenta a abertura de fissura, indicando um amolecimento. De forma esquemática essa relação pode ser descrita pela Figura 6a.

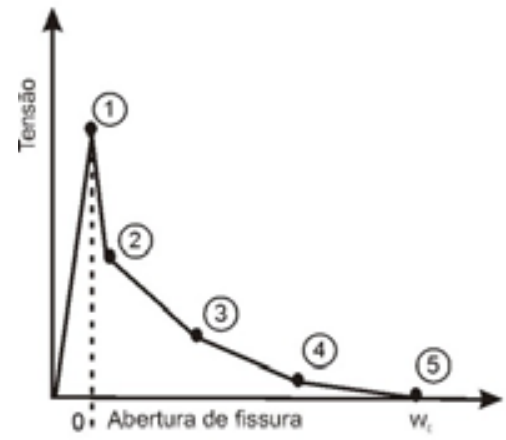

a)

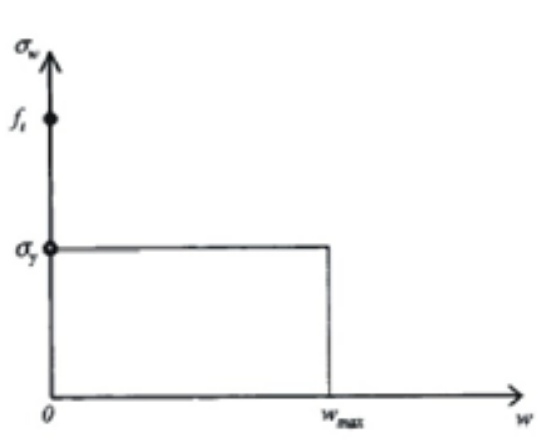

b)

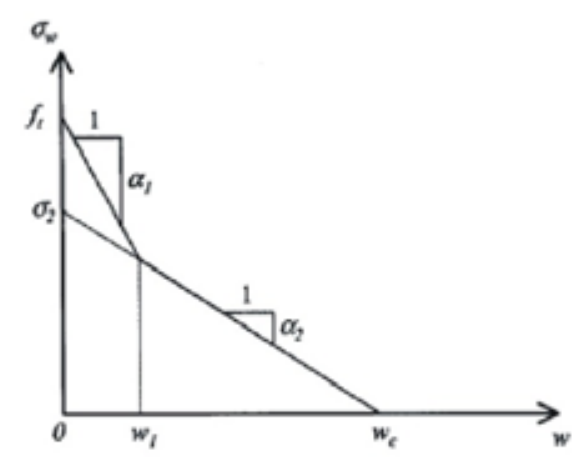

c)

Figura 6: a) Curva tensão-abertura esquemática para compósitos reforçado com fibra (Naaman e Reinhardt, 2006); b) modelo "drop-constant" (RILEM, 2002); c) modelo bi-linear (RILEM, 2002)

As tensóes correspondentes aos pontos $3 \mathrm{e}$ 4 podem ser maiores que a do ponto 2 , quando fibras de alto modulo ou quando grande volumes de fibras são utilizados. Para fibra curta de sisal dois comportamentos foram observados. Para compósitos reforçados com $1 \%$ de fibra, a tensão pós-fissuração mostrou-se constante, apresentando comportamento similar ao descrito por Soranakom e Mobasher (2007). Isso indica que as tensóes nos pontos 2, 3 e 4 são similares e iguais a uma tensão residual $s_{\mathrm{y}}$ que se mantém até que seja atingida a ruptura. Esse modelo, denominado 
"drop-constant" (RILEM, 2002), é apresentado na Figura $6 \mathrm{~b}$ e caracteriza materiais denominados dúcteis-frágeis (BAZANT, 2002).

Para volume de fibras de $2 \%$ ou $3 \%$, a tensão apresentou-se decrescente com o aumento da abertura da fissura, sendo esse comportamento, denominado quase-frágil por Bazant (2002) e é melhor descrito pelo modelo Bi-linear mostrado na Figura 6c (RILEM, 2002), adotando-se para $\mathrm{a}_{1}$ um valor igual a zero. A abertura de fissura $\mathrm{w}_{\mathrm{c}}$, mostrada na figura $6 \mathrm{a}$ (ponto 5), corresponde à tensão zero e indica que a abertura total da fissura foi atingida. Para os compósitos reforçados com fibras de sisal esse valor é dependente do tamanho da fibra e da sua inclinação cruzando a fissura, visto que a mesma é arrancada da matriz à medida que o deslocamento é aplicado.

$\mathrm{Na}$ Tabela 1 são listados os valores experimentais obtidos do ensaio de traçáo direta (Figura $3)$, considerando-se os modelos destacados nas Figuras 6 e 7. São apresentados também os valores da resistência e do módulo de elasticidade longitudinal obtidos do ensaio de compressão. Os valores de $\mathrm{w}_{\mathrm{c}}$ foram estimados, visto que o ensaio foi interrompido antes da ruptura do corpo de prova. Os valores entre parênteses correspondem ao coeficiente de variação em percentagem.

Tabela 1 - Resultados experimentais

\begin{tabular}{|c|c|c|c|c|c|c|}
\hline \multirow{2}{*}{ Mistura } & \multirow{2}{*}{$\mathrm{Vf}(\%)$} & \multicolumn{3}{|c|}{ Ensaio de Tração Direta } & \multicolumn{2}{c|}{ Ensaio de Compressão } \\
\cline { 3 - 7 } & & $\mathrm{f}_{\mathrm{t}}(\mathrm{MPa})$ & $\mathrm{s}_{\mathrm{y}} \mathrm{s}_{2}(\mathrm{MPa})$ & $\mathrm{w}_{\mathrm{c}}, \mathrm{w}_{\max }(\mathrm{mm})$ & $\mathrm{f}_{\mathrm{c}}(\mathrm{MPa})$ & $\mathrm{E}_{\mathrm{c}}(\mathrm{MPa})$ \\
\hline M1 & - & $3,00(3,7)$ & - & - & $42,71(0,4)$ & $26,17(9,1)$ \\
\hline MS1 & 1,00 & $2,58(15,6)$ & $0,28(20,0)$ & 6,0 & $41,60(8,8)$ & $25,34(2,1)$ \\
\hline MS2 & 2,00 & $2,59(22,3)$ & $0,74(23,8)$ & 6,0 & $27,18(4,9)$ & $24,88(1,8)$ \\
\hline MS3 & 3,00 & $2,79(21,8)$ & $1,22(13,4)$ & 7,0 & $32,19(6,4)$ & $21,58(1,4)$ \\
\hline
\end{tabular}

A partir da curva $\sigma$-w é possível obter informaçóes importantes para o dimensionamento de elementos estruturais. Obtida a função $\sigma(w)$ que descreve o comportamento pós-fissuração ou adotando um dos modelos da Figura 6, é possível representar o comportamento $\sigma$-w pela energia de fratura $G_{P}$, que pode ser calculada pela área sob a curva:

$$
G_{F}=\int_{0}^{w_{c}} \sigma(w) d w
$$

Uma quantidade que é derivada da energia de fratura é o comprimento característico $l_{\mathrm{ch}}$, que é definido como

$$
l_{c h}=\frac{E G_{F}}{f_{t}^{2}}
$$

onde E é o modulo de elasticidade e $\mathrm{f}_{\mathrm{t}}$ a resistência a tração.

O comprimento característico pode ser interpretado como a relação entre energia de fratura por área fissurada e a energia elástica por volume de um dado material. O comprimento $l_{\mathrm{ch}}$ mede a fragilidade do material: quanto maior o seu valor mais dúctil é o material. Na Tabela 2 são apresentados os valores experimentais de $\mathrm{G}_{\mathrm{F}}$ e $\mathrm{l}_{\mathrm{ch}}$ obtidos para os compósitos estudados, considerando a aplicação dos modelos teóricos às curvas experimentais mostradas na Figura 7.

Tabela 2-Parâmetros de fratura

\begin{tabular}{|c|c|c|}
\hline Mistura & $\mathrm{G}_{\mathrm{F}}\left(\mathrm{J} / \mathrm{m}^{2}\right)$ & $\mathrm{l}_{\mathrm{ch}}(\mathrm{mm})$ \\
\hline MS1 & 1680 & 6395,53 \\
\hline MS2 & 2220 & 8233,87 \\
\hline MS3 & 4270 & 11837,80 \\
\hline
\end{tabular}



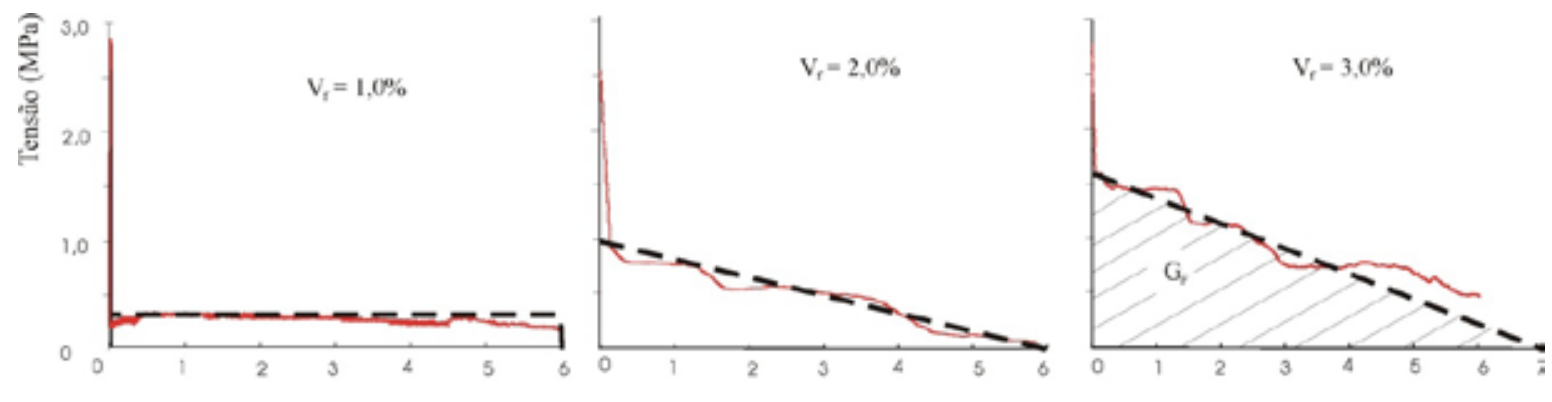

Figura 7. Modelagem das curvas tensão-abertura de fissura para os compósitos

Observa-se um aumento do valor de energia de fratura e comprimento característico com o aumento do teor de fibras de sisal, o que confirma o aumento da tenacidade do material. Para concretos com resistência à tração de 2,36 a 4,31 $\mathrm{MPa}$ tem sido encontrado valores de $\mathrm{G}_{\mathrm{F}}$ variando entre de 40 a $66 \mathrm{~J} / \mathrm{m}^{2}$ (Eliceset al, 2009). Para pasta de cimento e argamassa, Gustafsson (1985) encontrou valores de $G_{F}$ de $15 \mathrm{~J} / \mathrm{m}^{2}$ e $60 \mathrm{~N} / \mathrm{m}^{2}$, respectivamente. Isso indica que o compósito com fibra de sisal apresenta grande aumento da tenacidade de fratura, relativo aos materiais cimenticios não reforçados. É importante ressaltar, no entanto, que a abertura de fissura $w_{c}$ observada nos compósitos está fora da faixa aceitável para elementos construtivos de concreto, que é da ordem de 0 a 1,5 mm (RILEM, 2002), devido, principalmente, a questóes de durabilidade. Para concretos reforçados com teores de 20, 40 e $60 \mathrm{~kg} / \mathrm{m}^{3}$ de fibras de aço, Grossi (2006) encontrou valores de $G_{F}$ iguais a 1220,1400 e $3230 \mathrm{~J} / \mathrm{m}^{2}$, respectivamente, ao simular o comportamento sob tração direta. Esses valores se aproximam daqueles encontrados neste trabalho.

\section{CONCLUSÓES}

A partir da avaliação da adição de $1 \%, 2 \%$ e $3 \%$ de fibras curtas de sisal em uma matriz de argamassa de cimento verificou-se que o processo de fissuração do material sob tração é modificado. As fibras cruzando a fissura da matriz mantêm a continuidade de distribuição de tensôes ao longo da amostra, contribuindo assim para a redução da propagação da fissura e para a manutenção de uma tensão residual, mesmo após grandes deslocamentos.

Utilizando modelos analíticos baseados na Mecânica da Fratura foi possível avaliar a capacidade de absorção de energia do compósito fissura- do e conclui-se que tanto o valor da tensão pós-fissuração quanto da energia de fratura aumentam com o aumento do volume de fibra adicionado à matriz, tornando o material mais dúctil.

\section{REFERÊNCIAS}

BAZANT, Z.P. (2002) Concrete fracture models: testing and practice, Engineering Fracture Mechanics, Vol. 69, pp.165-205.

BRIBIÁN, I.Z.; CAPILLA, A.V.; USÓN, A.A. (2011) Life cycle assessment of building materials: comparative analysis of energy and environmental impacts and evaluation of the eco-efficiency improvement potential. Building and Environment, Vol. 46, pp.1133-1140.

CCI: Cement \& Concrete Institute (2010) Fibre Reinforced Concrete. Published by the Cement $\&$ Concrete Institute, Midrand.

COUTTS, R.S.P. (1988) Wood fibre reinforced cement composites. In: Natural fibre reinforced cement and concrete, V.5, Anais... Edited by R.N. Swamy, Blackie and Son Ltd, London, pp.1-62.

ELICES, M.; ROCCO, C.; ROSELLÓ, C.(2009) Cohesive crack modelling of a simple concrete: Experimental and numerical results. Engineering Fracture Mechanics, Vol. 76, pp.1398-1410.

FERREIRA, S. R. (2012) Influência da hornificação na aderência fibra-matriz e no comportamento mecânico de compósitos cimentíceos reforçados com fibras de sisal. Dissertaçao (Engenharia Civil e Ambiental). Universidade Estadual de Feira de Santana. 
GRAM, H.E. (1983) Durability of natural fibres in concrete, Swedish cement and concrete research institute, Research Fo.1:83, Stockolm.

GHEBRAB, T.T.; SOROUSHIAN, P. (2011) Mechanical properties of cement mortar: development of structure-property relationships. International Journal of Concrete Structures and Materials, Vol.5, pp.3-10.

GUSTAFSSON, P. J. (1985) Fracture mechanics studies of non-yielding materials like concrete: modeling of tensile fracture and applied strength analysis. LundInstituteof Technology, Report TVBM-1007, Lund, Sweden.

GROSSI, B.F. (2006) Uma contribuição a modelagem numérica ao concreto com fibras curtas de aço. Tese (Engenharia de Estruturas). Universidade Federal de Minas Gerais.

LI, V.C., MISHRA. D.K., WU, H. (1995) Matrix design for pseudo-strain-harding fibre reinforced cementitious composites, Materials and Structures, V.28, p. 586-595.

LIMA, P.R.L. (2004) Análise teórica e experimental de compósitos reforçados com fibras de sisal. Tese (Engenharia Civil). Universidade federal do Rio de Janeiro.

LIMA, P.R.L.; TOLEDO FILHO, R. D. (2008) Uso de metacaulinita para incremento da durabilidade de compósitos à base de cimento reforçados com fibras de sisal, Ambiente Construido, Vol.8, N.4, pp.7-19.

NAAMAN, A.E. (2012) Half a century of progress leading to ultra-high performance fiber reinforced concrete: Part 1-overall review. In: 2th International RILEM Conference on Strain Hardening Cementitious Composites. Rio de Janeiro, p.17-26.

NAAMAN, A.E., REINHARDT, H.W. (2006) Proposed classification of HPFRC composites based on their tensile response. Materials and Structures, Vol.39, pp.547-555.

NORTON, B; SKATES, H. (2000) Technologies for sustainable buildings. In: World Renewable Energy Congress VI. Ed. Elsevier.

RILEM TC 162-TDF (2002) Test and design methods for steel fibre reinforced concrete, Matériaux et Constructions, Vol.35, pp.262-278.

SORANAKOM, C.; MOBASHER, B. (2007) Closed-form solutions for flexural response of fiber-reinforced concrete beams, Journal of Engineering Mechanics, Vol.133, N.8, pp.933-941.

STANG, H.; LI, V.C., KRENCHEL, H. (1995) Design and structural applications of stress-crack width relations in fibre reinforced concrete, Materials and Structures, Vol.28, pp.210-219.

TOLÊDO FILHO, R. D. (1997) Natural fibre reinforced mortar composites: experimental characterization. Tese (Doutorado em Engenharia Civil). Pontificia Univerisdade Católica do Rio de Janeiro.

TOLEDO FILHO, R.D.; GHAVAMI, K.;ENGLAND, G.L; SCRINEVER, K. (2003) Developmentofvegetablefibre-mortarcompositesofimproveddurability. Cement and Concrete Composites, Vol.25, N.2, pp.185-196.

VAN MIER, J.G. (1997) Fracture processes of concrete: assesment of material parameters for fracture models. CRC Press.

VISALVANICH, K., NAAMAN, A.E. (1983) Fracture model for fiber reinforced concrete, $A C I$ Materials Journal, pp.128-38.

VONK, R.A. (1993) A micromechanical investigation of softening of concrete loaded in compression, Heron, Vol. 38, N.3. 